\title{
Numerical analysis of thermal radiation of spacecraft on orbit based on SINDA/FLUINT
}

\author{
Yi SHI ${ }^{1, *}$ \\ ${ }^{1}$ Institute of Thermal Science Technology, Shandong University, Jinan 250061, China
}

\begin{abstract}
Thermal stability is an important indicator to measure the reliability of aerospace equipment, and the space thermal environment is complex, and the commonly used thermal analysis software for ground equipment can no longer meet the needs. For this reason, based on the thermal design analysis software SINDA/FLUINT, taking a spacecraft as the research object, the thermal balance equation is first established. Then, use the thermal desktop software to establish a numerical model, and use the SINDA/FLUINT software to solve the heat transfer process. Finally, the thermal desktop is used to post-process the calculation results and analyse the thermal radiation of the spacecraft.
\end{abstract}

\section{Introduction}

On September 21, 1992, the Chinese government decided to implement the manned space project and decided on a three-step development strategy. On June 12, 2019, China Manned Space Engineering Office and the United Nations Office for Outer Space Affairs jointly announced in Vienna that nine projects of 17 countries have been selected into the first batch of scientific experiments of Chinese Space Station. With the rapid development of the national aerospace industry, the performance and operating life of spacecraft will surely become the cornerstone of the development of the aerospace industry.

After the spacecraft is launched into space, the environment of the space target is equivalent to a radiation source with infrared characteristics. With the rapid development of infrared technology, the use of infrared radiation characteristics to detect space targets is an effective method1. Faced with this situation, the common analysis methods conclude formula derivation, model simplification, solving the heat balance equation of nodes, space target temperature distribution calculation, and then according to the surface material temperature distribution and emissivity to solve the infrared radiation characteristics of space target. This method is easy to understand, but different calculation models need to be established for different targets in different environments. The program design has a large amount of engineering, and it has no universality and poor adaptability 6 . While the numerical simulation method does not need to simplify and assume the target. Compared with the traditional calculation method, it has the advantages of high calculation accuracy, strong real-time performance, not limited by the target structure, and strong operability. It makes up for the shortcomings of the traditional method, such as long time-consuming, poor accuracy, and many constraints. It is an effective method for the thermal radiation analysis of complex space targets 7 .

On the basis of previous studies, this paper takes deep space as the research background and spacecraft as the research object. Firstly, the main radiation sources on the surface of spacecraft are analysed, and the thermal balance equation of the spacecraft is established. Then, the spacecraft model is built, and the thermal grid of the model is generated. Finally, after setting the boundary conditions, the real-time thermal balance simulation of spacecraft is completed by using SINDA/FLUINT solver.

\section{Thermal Desktop and SINDA/FLUINT}

SINDA/FLUINT is a comprehensive finite difference and lumped parameter software for thermal design analysis and fluid flow analysis of complex systems. SINDA/FLUINT is a comprehensive finite difference software for thermal design analysis and fluid flow analysis of complex systems, developed by Cullimore \& Ring, USA8. SINDA/FLUINT has provided the most reliable heat transfer and fluid flow design and analysis services in the aerospace industry for many years. SINDA/FLUINT was awarded the "American Aerospace Contribution Award" in 1991 for its outstanding performance in the industry9.

Thermal desktop is based on the AutoCAD software environment. Users can directly use the existing geometric or FEM models, or use these models as reference. Through "automatically grabbing key points", they can quickly generate the finite element, surface and entity of thermal desktop, and complete the thermal model design and pre-processing and post-processing.

RadCAD software uses Monte Carlo ray tracing theory to calculate the angle coefficient, radiative heat conduction and heat flux between quadric surfaces. The

* Corresponding author: yi.s@sdu.edu.cn 
calculation results can be directly imported into SINDA/FLUINT software.

\section{Analysis of thermal environment of spacecraft}

In the whole life cycle of a space target, it will be affected by the thermal environment during launch and on orbit operation. Among them, the thermal environment has the greatest impact on the space target during its on-orbit operation. In addition to the heat dissipation of its own electronic equipment, the space object in orbit is also affected by the orbital external heat flow. The space target can only exchange heat through heat conduction and radiation, which is in the space environment.

\subsection{Analysis of heat radiation exchange}

When the spacecraft is in the earth orbit as a whole, the way of heat exchange with the external environment is almost radiation heat transfer, and there is no convective heat transfer and heat conduction. While the spacecraft is in orbit, it will receive the solar radiation from the sun, the infrared radiation of the earth and the solar radiation reflected by the earth10. In addition, the spacecraft will dissipate its own heat into space, as shown in Fig. 1.
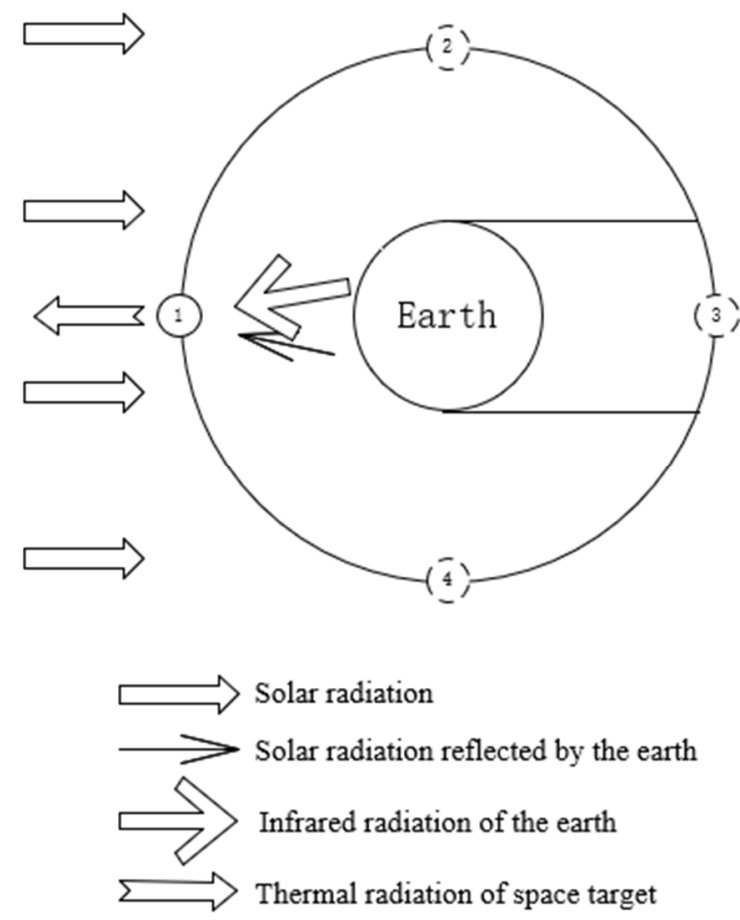

Fig. 1. On orbit operation diagram of spacecraft

According to the law of conservation of energy, the heat balance equation of spacecraft in orbit can be worked out. From Fig. 1, it is obvious that the radiation received by the spacecraft includes solar radiation, solar radiation reflected by the earth, infrared radiation of the earth, the heat transfer of internal heat source of the spacecraft. In unit time, the sum of the heat absorbed by the space target from the space and the heat generated by the space target is equal to the sum of the heat emitted by the space target to the space and the change of the internal energy of the space target. Therefore, the sum of the heat fluxes of the above four parts should be equal to the heat flux radiated by the spacecraft to the universe and the change of its internal energy.

\subsection{Solving process}

A spacecraft can be roughly regarded as a grey body. In order to analyse its thermal radiation characteristics, the surface heat flux and surface temperature must be calculated, and the emissivity of the surface material must be obtained. The surface heat flux and temperature distribution mainly depend on the heat exchange and heat balance between the surrounding environment and the spacecraft; the emissivity depends on the type of target surface material. The steps for analysing the thermal radiation of spacecraft based on SINDA/FLUINT are as follows:

(1) Build the spacecraft model based on the geometry and material properties of the spacecraft by using Thermal Desktop;

(2) Perform thermal meshing on the established spacecraft model, establish boundary nodes and set boundary conditions based on its thermal load information;

(3) Set the orbital parameters of the spacecraft;

(4) Use SINDA/FLUINT software to perform on-orbit thermal radiation calculations of the spacecraft thermal model;

(5) Analyze the thermal radiation of the spacecraft in orbit based on the calculation results.

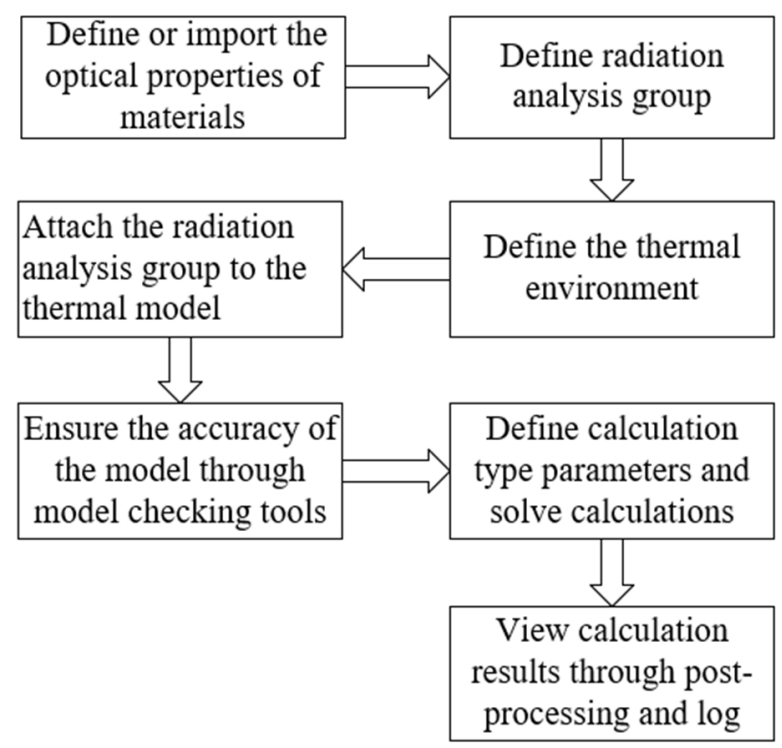

Fig. 2. Flow chart of numerical simulation of thermal radiation analysis

\subsection{Theoretical calculation model}

Due to the different temperatures of various parts of the spacecraft during the actual flight, there is a phenomenon of heat conduction inside the spacecraft. Therefore, when 
establishing the thermal balance equation of the microelement area according to the node network method, it is necessary to consider the heat conduction between the nodes in the component. According to 3.1, according to the node network method, combined with the thermal environment of the spacecraft, the thermal balance equation of any node $i$ on the spacecraft is:

$$
Q_{1}+Q_{2}+Q_{3}+Q_{4}+Q_{5}+Q_{i}=Q_{6}+Q_{7}
$$

where the $Q_{i}$ is the internal heat source.

The $Q_{1}, Q_{2}, Q_{3}$ are solar radiation, solar radiation reflected by the earth, and the infrared radiation of the earth:

$$
\begin{aligned}
& Q_{1}=\alpha_{s} S \phi_{s} A_{i} \\
& Q_{2}=\alpha_{s} E_{r} \phi_{r} A_{i} \\
& Q_{3}=\varepsilon_{e} E_{i} \phi_{i} A_{i}
\end{aligned}
$$

where the $\alpha_{\mathrm{s}}$ is the solar absorption rate of the external surface of each node, $S$ is the solar constant, $A_{i}$ is the area of node $i . \phi_{s}, \phi_{r}, \phi_{i}$ are the geometrical angle coefficients of solar radiation, the solar radiation reflected by the earth and the infrared radiation of the earth of node $i$ respectively. $E_{r}$ is the average reflection density of the surface of the earth to solar radiation, $E_{i}$ is the average infrared radiation density on the surface of the earth.

The $Q_{4}$ is the part of the energy absorbed by the surface $A_{i}$, which is emitted by the entire spacecraft surface :

$$
Q_{4}=\sum_{j=1}^{n} B_{i, j} A_{j} \varepsilon_{j} \sigma T_{j}^{4}
$$

where the $B_{i, j}$ is the absorption factor of node $i$ to node $j$, $\varepsilon_{j}$ is the emissivity of node $j, \sigma$ is the Stefan-Boltzmann constant, and the value is $5.66 \times 10^{-8} \mathrm{~W} /\left(\mathrm{m}^{2} \cdot \mathrm{K}^{4}\right), T_{j}$ is the thermodynamic temperature of node $j$.

The $Q_{5}$ is the amount of heat conduction between $A_{i}$ and other nodes :

$$
Q_{5}=\sum_{j=1}^{n} K_{j, i}\left(T_{j}-T_{i}\right)
$$

where the $K_{j, i}$ is the conduction factor between node $j$ and $i$, which depends on the geometric parameters of the conduction path, the thermal conductivity of the material and the thermal conductivity of the contact between the surfaces, etc.

The $Q_{6}$ and $Q_{7}$ represent the change of the heat radiated outward by $A_{i}$ and the change of the internal energy of $A_{i}$ respectively :

$$
\begin{gathered}
Q_{5}=A_{i}\left(\varepsilon_{1 i}+\varepsilon_{e i}\right) \sigma T_{i}^{4} \\
Q_{6}=m_{i} c_{i} \frac{\Delta T_{i}}{\Delta \tau}
\end{gathered}
$$

where the $\varepsilon_{l i}, \varepsilon_{e i}, m_{i}$ and $c_{i}$ are the emissivity of the inner and outer surfaces of node $i$, mass and specific heat capacity of node $i$.

\subsection{Numerical Simulation}

Take a spacecraft as the research object, use Thermal Desktop software to establish the orbit of the spacecraft and define the relevant orbital parameters:

Table 1. The parameters of orbit.

\begin{tabular}{|c|c|}
\hline Name & Parameters \\
\hline Altitude & $500 \mathrm{Km}$ \\
\hline Beta angle & 90 degrees \\
\hline Albedo & 0.87 \\
\hline Solar flux & $0.8735 \mathrm{~W} / \mathrm{in}^{2}$ \\
\hline Position & 90 (only) \\
\hline
\end{tabular}

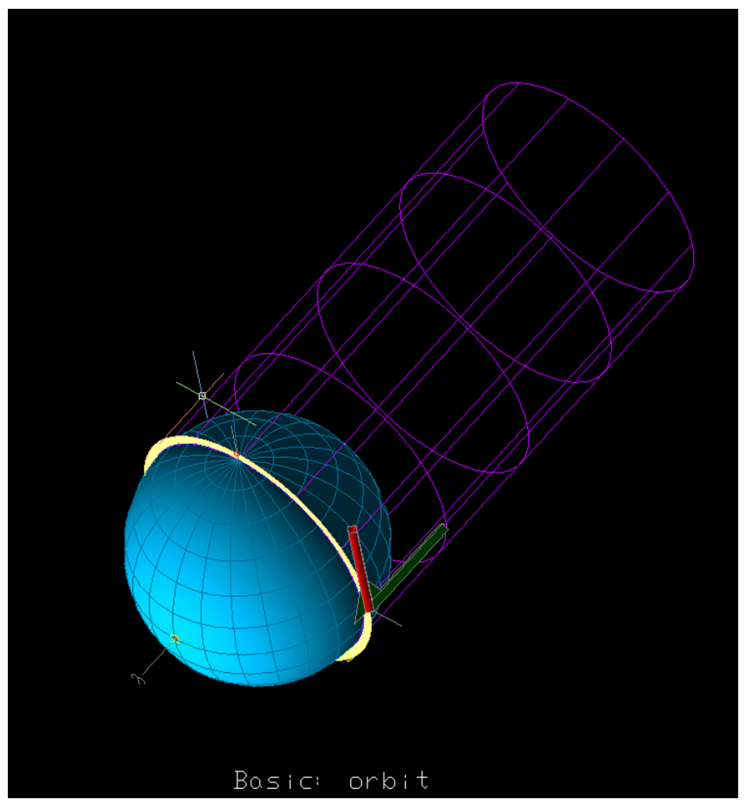

Fig. 3. Spacecraft orbit diagram

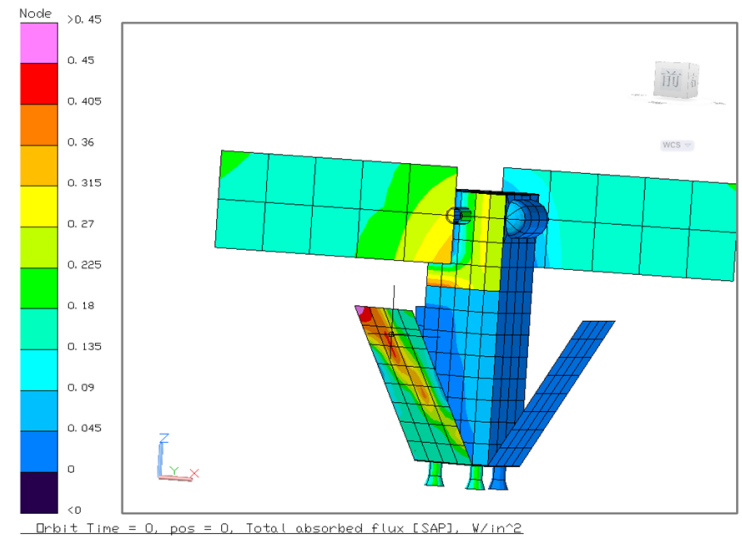

Fig. 4. External heat flux of spacecraft 


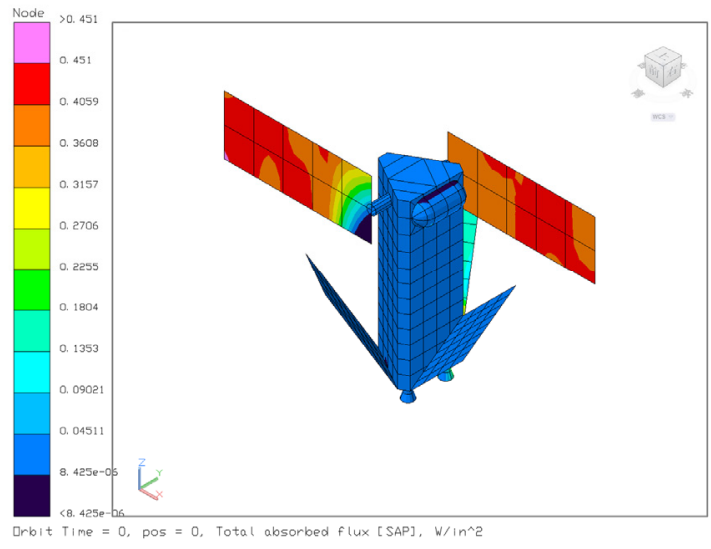

Fig. 5. External heat flux of spacecraft with $\mathrm{X}$ additional rotation set as 180

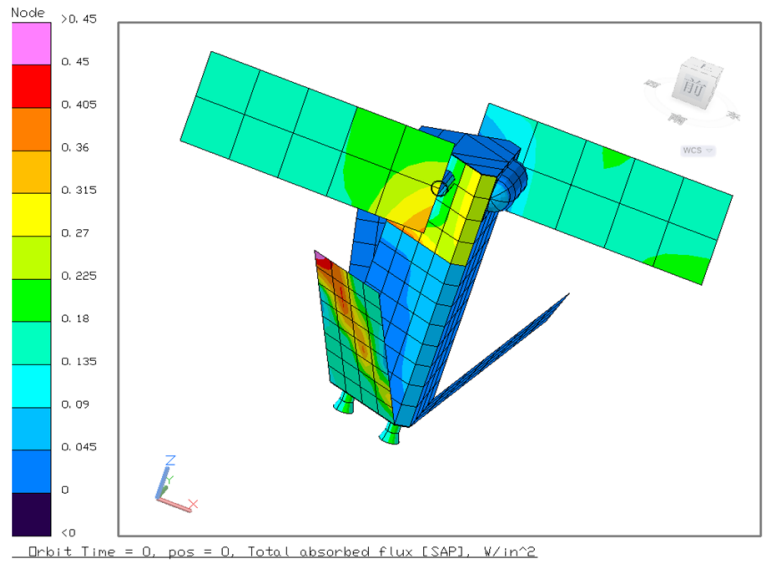

Fig. 6. External heat flux of spacecraft with Y additional rotation set as 180

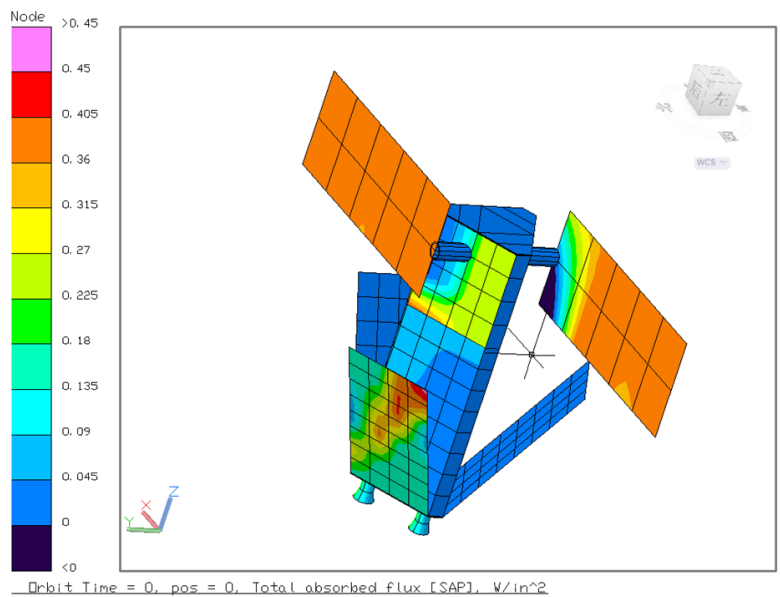

Fig. 7. External heat flux of spacecraft with $\mathrm{Z}$ additional rotation set as 180

In the process of numerical simulation, the spacecraft is set in a fixed position. Other conditions remain unchanged, by adjusting the rotation angle of the spacecraft's own coordinate system, a group of the total heat flux distribution map of the spacecraft are obtained.

Fig. 4 shows the heat flux distribution of the spacecraft without adjustment. And Fig. 5, Fig. 6 and Fig. 7 show the heat flux distribution of the spacecraft after rotating 180 degrees around its own axis $\mathrm{X}, \mathrm{Y}$ and $\mathrm{Z}$ only, respectively.
When the spacecraft is not adjusted, the main heating part of the spacecraft is the front of the spacecraft, and the lower part is heated more than the upper part. As can be seen from Fig. 5 and Fig. 7, when the spacecraft rotates $180^{\circ}$ along its own coordinate axis $\mathrm{X}$ or $\mathrm{Z}$, at this position, the upper part of the spacecraft becomes severely heated, and in the case of additional rotation set as 180, there are more heated elements on the upper parts of spacecraft than that with $\mathrm{X}$ additional rotation set as 180 . Among them, the main heated part of the spacecraft with $Z$ additional rotation set as 180 is the back side of it.

Fig. 8, Fig. 9 and Fig. 10 are the radiant heat flow images of the spacecraft with $\mathrm{X}, \mathrm{Y}$ and $\mathrm{Z}$ additional rotation set as 90 respectively.

It can be seen from Fig. 8 and Fig. 10 that after the spacecraft rotates $90^{\circ}$ around its $\mathrm{X}$ axis, the main heated part of the spacecraft is transferred from the front to the top, which is shifted from the front to the left when the spacecraft rotates the same degree around its $Z$ axis. While rotating around its $\mathrm{Y}$ axis at the same position has no effect on the spacecraft.

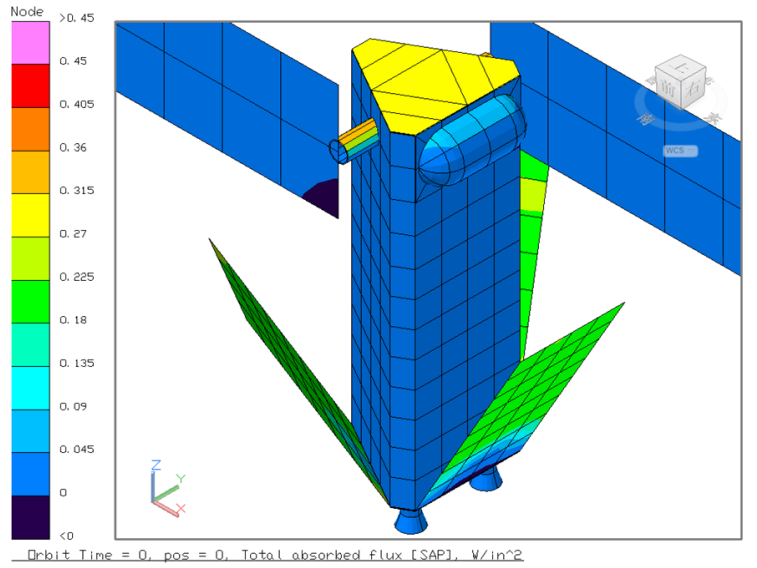

Fig. 8. External heat flux of spacecraft with $X$ additional rotation set as 90

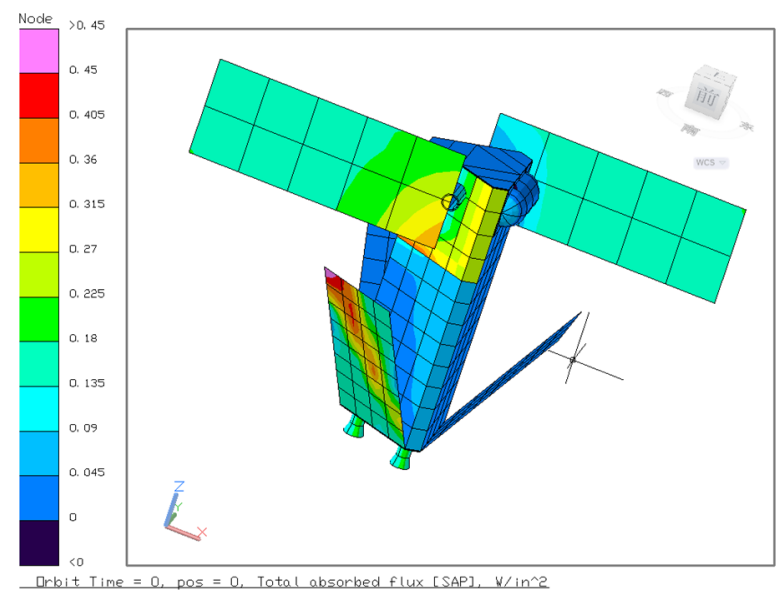

Fig. 9. External heat flux of spacecraft with $Y$ additional rotation set as 90 


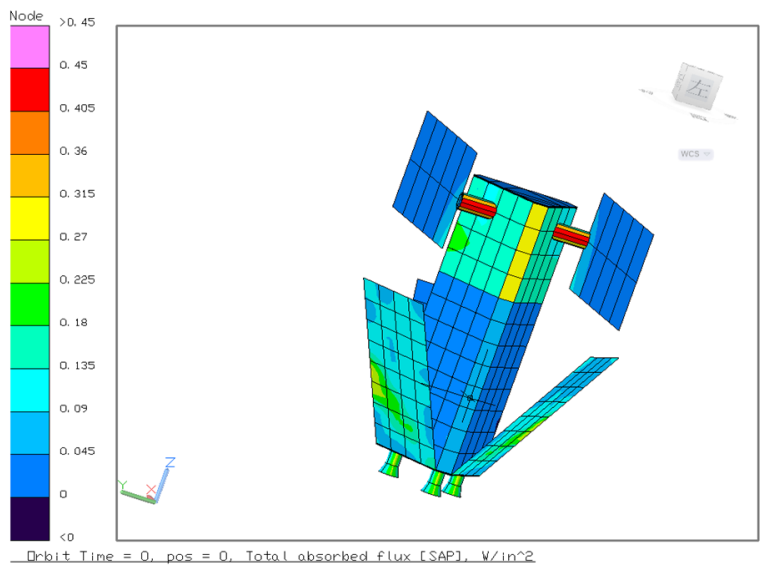

Fig. 10. External heat flux of spacecraft with $\mathrm{Z}$ additional rotation set as 90

\section{Conclusion}

For the space targets in special environments, numerical simulation is one of the most commonly used research methods. This paper used SINDA/FLUINT software to perform the numerical simulation of spacecraft, and analysed the thermal radiation of the spacecraft on orbit. Numerical simulation can not only be used to simulate and analyse the thermal environment of space targets, but also can carry out a systematic geothermal analysis of space targets without restriction, so as to better design and optimize the space target.

Compared with traditional calculation methods, numerical simulation method has the advantages of high calculation accuracy, strong real-time performance, less restriction, and strong operability. It makes up for the shortcomings and shortcomings of traditional methods such as long time-consuming, poor accuracy, and many limiting factors. It is an effective method for thermal radiation analysis of complex space targets.

\section{References}

1. Bot. C, G.Helou, et. Serendipity Observations Of Far Infrared Cirrus Emission In The Spitzer Infrared Nearby Ga,axies Survey: Analyses Of Far-Infrared Correlations[J]. Astrophysical Journal, 2009, 695(1): 469-478

2. Shen, T. S., M. Guo, et. IR image generation of space target based on openGL[C]. 5th International Symposium on Multispectral Image Processing and Pattern Recognition, Wuhan, PEOPLES R CHINA, 2007, 67865A:1-5.

3. Wood, K. Infrared signatures and models of circumstellar dust disks[J]. New Astronomy Reviews, 2008, 52(2-5): 145-153.

4. Bot. C, G.Helou, et. Serendipity Observations Of Far Infrared Cirrus Emission In The Spitzer Infrared Nearby Ga, axies Survey: Analyses Of Far-Infrared Correlations[J]. Astrophysical Journal, 2009, 695(1): 469-478.
5. F. Zhang, F. Xiang, et. Theoretical analysis of infrared radiation of space target[J]. Infrared and Laser Engineering, 2007(S2):419-422.

6. Z. Wang, Y. Zhao, et. IR Analysis of Space Target Based on Sinda/Fluint[C]. 20th National Conference on Computer Science and Technology Application, 2009:695-699.

7. X. Kong, S. Liao, et. Numerical Simulation of the Space Target Surface Temperature Filed Based on Sinda/Fluint[J]. Infrared Technology, 2012, 34(10): 580-583.

8. Sinda/Fluint Users Manual[EB/OL]. [2009-4-10]. http://www.crtech.com.

9. Mark J.Welch and Tim Panczak. Automating Thermal Analysis with Thermal Desktop[C]// 29th ICES, SAE paper 1999, 01: 2156.

10. G. Min, S. Guo. Satellite thermal control technology [M]. Beijing:The Since Publishing House, 1998 :7074. 